\title{
The coordinated regulation of mitochondrial structure and function by Drp1 for mitochondrial quality surveillance
}

\author{
Hyo Min Cho \& Woong Sun* \\ Department of Anatomy, Korea University College of Medicine, Brain Korea 21 Plus, Seoul 02841, Korea
}

\begin{abstract}
Mitochondrial morphology is known to be continuously changing via fusion and fission, but it is unclear what the biological importance of this energy-consuming process is and how it develops. Several data have suggested that mitochondrial fission executed by Drp1 is necessary to select out a damaged spot from the interconnected mitochondrial network, but the precise mechanism for the recognition and isolation of a damaged sub-mitochondrial region during mitochondrial fission is yet unclear. Recently, Cho et al. found that the mitochondrial membrane potential (MMP) is transiently reduced by the physical interaction of Drp1 and mitochondrial Zinc transporter, Zip1, at the fission site prior to the typical mitochondrial division, and we found that this event is essential for a mitochondrial quality surveillance. In this review, Cho et al. discuss the role of a mitochondrial fission in the mitochondrial quality surveillance system. [BMB Reports: Perspective 2019; 52(2): 109-110]
\end{abstract}

Mitochondria are considered to be the all-around players responsible for the generation of cellular energy, and leading up to the induction of apoptosis. These identified missions of the mitochondria are essential for life, and the incidence of a

*Corresponding author. E-mail: woongsun@korea.ac.kr

https://doi.org/10.5483/BMBRep.2019.52.2.032

Received 20 January 2019

Keywords: Drp1, Mitochondria, Mitochondrial fission, Mitochondrial membrane potential, Zip1

Abbreviations: CCCP, Carbonyl cyanide m-chlorophenylhydrazone; Drp1, Dynamin-related protein 1; DRPs, Dynamin-related proteins; MCU, Mitochondrial calcium uniporter; Mfn1/2, Mitofusin 1/2; MIS, mitochondrial intermembrane space; MMP, Mitochondrial membrane potential; MOM, mitochondrial outer membrane; MPP, Mitochondrial processing peptidase; OPA1, Optic atrophy 1; PARL, Presenilin-associated rhomboid-like protease; PINK1, PTEN-induced putative kinase 1; ROS, Reactive oxygen species

Perspective to: Cho HM et al., 2019, Drp1-Zip1 interaction regulates mitochondrial quality surveillance system, Molecular Cell, 73(2), p364-376, doi: 10.1016/j.molcel.2018.11.009 mitochondrial malfunction is often associated with many health problems in humans, including neurodegenerative disease and diabetes. Thus, while mitochondria are meant to be utilized especially for the production of cellular energy, cells also need to invest large resources to maintain and keep the mitochondria healthy. For instance, mitochondria continuously change their morphology through the energy-consuming fusion and fission processes, which are regulated by DRPs including Mfn $1 / 2$, Opa1, and Drp1. This dynamic change of mitochondrial morphology is closely linked with the mitochondrial quality control, including the process of mitophagy. For example, the process of fusion of impaired mitochondria with healthy ones at least partially restores the function of damaged mitochondria. On the other hand, the process of fission can segregate damaged sections from normal mitochondria, and allow damaged mitochondria to be removed by the process of mitophagy. Thus, mitochondrial fission can be a mechanism to remove damaged regions in the mitochondrial network, and serve to prevent mitochondrial dysfunction by damage accumulation (Pickrell AM and Youle RJ (2015) Neuron 85, 257-273).

In an effort to be consistent with this model, the treatment with the mitochondrial uncoupler, such as CCCP, induces global dysfunction of the mitochondria and subsequently triggers Drp1-dependent mitochondrial fragmentation and the mitophagy phase. It is known that CCCP drastically reduces MMP, which inhibits the uptake of calcium into the mitochondria, and subsequently causes a transient increase in the calcium concentration around the mitochondria. This increased calcium activates calcineurin, which will work to develop a process causing mitochondrial fragmentation through dephosphorylation of Drp1. On the other hand, the PINK1 that is cleaved in MIS via MPP and PARL, at that point becomes stabilized at MOM when the MMP is reduced, and it recruits and phosphorylates the E3 ubiquitin ligase Parkin. Activated parkin ubiquitinates mitochondrial proteins and the PINK1 phosphorylates ubiquitin-labeled proteins, which recruits mitophagic receptors to proceed to the phase of mitophagy (Bingol B and Seng M (2016) Free Radic Biol Med 100, 210-222). In this model, the MMP reduction acts at the upstream of the Drp1 and Parkin recruitments, and initiates both mitochondrial fission and mitophagy. The prevention of Drp1 activity mitigates the mitophagy, suggesting that the mitochondrial fission which is then reduced to a bite sized form, is necessary for the efficient autophagic engulfment. 
Other lines of evidence suggest that modifying the activities of the mitochondrial fission/fusion proteins affect the MMP. For example, the case of the overexpression of Mfn2 increases mitochondrial fusion, and these elongated mitochondria exhibit high MMP. Inhibition of Mfn $1 / 2$ or OPA 1 conversely induces mitochondrial fragmentation, and the decreased mitochondrial respiration (Pich S et al. (2005) J Cell Biol 14, 1405-1415; Loiseau D et al., (2007) Ann Neurol 61, 315-323). Likely, it is the case that the Drp1 overexpression induces mitochondrial fragmentation with a reduction of the MMP. In the meantime, a blockage of the mitochondrial fragmentation by the Mdivi-1 (an inhibitor of Drp1) treatment prevents a MMP reduction and a decrease in the apoptosis upon the process of oxidative stress (Sarin $\mathrm{M}$ et al. (2013) Cell Death Dis 4, e670; Ong SB et al., (2010) Circulation $121,2012-2022)$. By the same token, the knockdown of the Drp1 receptors, such as Fis1 and Mff, also inhibits mitochondrial fragmentation and MMP loss, which is therefore ultimately more resistant to the process of mitophagy (Gao F et al., (2015) Hum Mol Genet 24, 2528-2538). Therefore, it appears that mitochondrial fission/fusion status can affect the mitochondrial MMP level, but the precise molecular mechanism regarding how these mitochondrial fission machinery and MMP levels are coordinated is yet unclear.

Cho et al. recently reported that the MMP is transiently and focally reduced at the mitochondrial fission site, which is dependent on the physical interaction of Drp1 with a newly identified mitochondrial zinc transporter, Zip1. Because the Zip1 is also associated with MCU to allow a zinc ion delivery from the cytosol to the mitochondrial matrix, the Drp1-Zip1 interaction reduces the MMP by increasing the mitochondrial zinc. Because a physical interaction occurs immediately after the recruitment of Drp1 on the mitochondria, the MMP reduction on the mitochondrial fission site is seen to occur several seconds prior to the completion of the mitochondrial fission. This time-lag is known to be owing to the time interval identified between initial the Drp1 recruitment and the

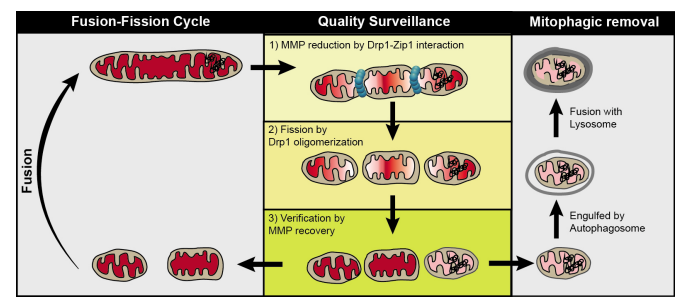

Diagram 1. Schematic illustration for the Drp1-Zip1 interactiondependent mitochondrial quality surveillance during the mitochondrial fission. Mitochondrial morphology continuously changed by the fusion-fission cycle. (1) Upon stimuli, Drp1 moves to the mitochondria, and interacts with the mitochondrial Zip1 to induce the focal MMP reduction. (2) Drp1 cuts the mitochondria through their oligomerization. (3) Healthy piece of mitochondria recovers their MMP and stays in the fusion-fission cycle, but the damaged mitochondria fail to recover and are subsequently removed by the mitophagy. execution of mitochondrial fission (Ji WK et al. (2015) Elife 4, e11553). Importantly, the Drp1-Zip1 interaction does not require the GTPase activity of the Drp1, and it does not affect the activity of the Drp1-dependent mitochondrial fission. Rather their interaction induces mitochondrial zinc entry presumably via activation of Zip1, although the precise mechanism how the Zip1-mediated zinc entry is enhanced by the Drp1 interaction.

Interestingly, the reduction of the MMP during mitochondrial fission was soon recovered in most healthy mitochondria, and they appear to stay in the pool of the mitochondrial fusion/fission cycle. On the other hand, some of the mitochondria failed to do so, and were subsequently tagged by the mitophagy process. Thus, the prolonged inhibition of the Drp1-Zip1 interaction caused an accumulation of impaired mitochondria and subsequently disfavored the health/growth of the cells as hallmarked by the reduced ATP production, increased mitochondrial ROS, and the retardation of neuronal growth. In conclusion, the study provided a molecular mechanism regarding how the Drp1-dependent mitochondrial fission contributes to the removal of damaged sectors in the mitochondrial network, to maintain mitochondrial quality. Drp1-dependent MMP reduction appears to be a quality examiner, and only mitochondria which can recover the Drp1-induced stress (MMP reduction) can be maintained in the fusion-fission cycle. Thus, these results suggest that the Drp1 plays dual roles to identify (MMP reduction by Zip1 interaction) and to isolate (fission by self-oligomerization) the damaged mitochondria for efficient removal (Diagram 1).

This new model may explain how the energy-consuming mitochondrial fusion-fission cycle serves for the quality surveillance of mitochondria in the physiological condition. However, it is also true that there are several issues to validate this tempting hypothesis. It is yet unclear whether the recruitment of the Drp1 on the mitochondria is considered a random event, or can be marked by any events triggered by the identified mitochondrial impairment. Considering that CCCP can promote the Drp1 activation and recruitment, it is plausible that a sub-threshold reduction in the mitochondrial impairment may prime the recruitment of Drp1 on the damaged spot. What is the specific mechanism to modify Zip1 activity by the Drp1 interaction? What is the role of zinc entry for the MMP reduction? It is also interesting to explore how the MMP reduction can be subsequently recovered: Is the de-novo synthesis from the mitochondrial gene involved in the recovery? In other words, are the presence/absence or the mutation in the mitochondrial genome associated with the fate of the mitochondrial fragments after the fission? Exploration of these and related questions will nourish the model.

\section{ACKNOWLEDGEMENTS}

This research was supported by the National Research Foundation of Korea (NRF-2017M3A9B3061308 and NRF2018R1A2A3075271). 